\title{
On the effect of tip vortices in low-Reynolds-number post-stall flow control*
}

\author{
Kunihiko Taira $^{\dagger}$ and Tim Colonius ${ }^{\ddagger}$ \\ California Institute of Technology, Pasadena, California, 91125
}

\begin{abstract}
We numerically investigate the application of steady blowing to three-dimensional stalled flows around low-aspect-ratio rectangular flat-plate wings at a Reynolds number of 300 . The objective of this study is to explore techniques to enhance lift by directly modifying the dynamics of the wake vortices. Out of various combinations of forcing location and direction considered, we identify two configurations that provide significant lift enhancement. In these cases, actuation appears to strengthen the tip vortices for increased downward induced velocity upon the leading-edge vortices. This in turn moves the low-pressure core directly above the top surface of the wing to greatly enhance lift.
\end{abstract}

\section{Introduction}

With the ability to provide close surveillance in dense geography or urban areas, micro air vehicles have been of great interest to the military and civilian communities. Due to their unique size and operational environment, a wide variety of vehicle designs are considered with rigid, ${ }^{1}$ flapping, ${ }^{2}$ rotating, ${ }^{3}$ and flexible ${ }^{4}$ wings. In all cases, the use of low-aspect-ratio wings in low-Reynolds-number flights has prompted the need for further research as the flow physics is different from the classical high-aspect-ratio, high-Reynoldsnumber aerodynamics (see Refs. 5-7). For the current investigation, we focus on low-aspect-ratio wings in pure translation.

A number of studies have been carried out to understand and characterize the low-Reynolds-number aerodynamics around purely translating low-aspect-ratio wings. ${ }^{8-12}$ The experiments by Torres and Mueller ${ }^{11}$ have characterized the aerodynamic performance of various low-aspect-ratio wings at Reynolds numbers $(R e)$ of $\mathcal{O}\left(10^{5}\right)$. Such flows are found to be vastly different from those at high Reynolds numbers around high-aspect-ratio wings due to the viscous effects and the three-dimensionality. A comprehensive list of experimental and numerical studies of the aerodynamics for micro air vehicles is compiled in Ref. 5. More recently, Taira and Colonius ${ }^{13}$ have studied the three-dimensional separated flows behind low-aspect-ratio wings at post-stall angles of attack at $R e=\mathcal{O}(100)$ with emphasis on the vortex dynamics. It was observed that the tip vortices have significant influence on the wake structures and the corresponding forces exerted on the wings.

At post-stall angles of attack, wings experience reduced lift due to flow separation. However, specific arrangements of the wake vortices are known to provide enhanced aerodynamic performance. For example, insects are known to benefit from the wake vortices. The stable attachment of the leading-edge vortices provides added spanwise circulation, yielding large increase in lift. ${ }^{14-18}$ Inspired by how animals favorably use vortical forces, we investigate the application of steady blowing to alter the dynamics of the wake vortices behind low-aspect-ratio wings to achieve lift enhancement (direct wake modification ${ }^{19}$ ). Our objective is not to reattach the flow or suppress the unstable modes in the wake.

In addition to the unique wing designs and the flight Reynolds number, micro air vehicles operate under large wing gusts. ${ }^{20}$ Hence, flow control becomes a key requirement for the vehicle to provide stable performance. Past investigations have employed periodic excitation ${ }^{21-23}$ to delay airfoil stall. Such excitation

\footnotetext{
*Accepted for publication in the AIAA Journal (December 5, 2008).

${ }^{\dagger}$ Graduate Research Assistant, Mechanical Engineering; Currently Postdoctoral Research Associate, Mechanical and Aerospace Engineering, Princeton University. Member AIAA.

†Professor, Mechanical Engineering. Senior Member AIAA.
} 
is introduced to trigger inherent instabilities in the flow to alter the wake in a favorable manner, i.e., to reattach the flow or delay separation.

Here we conduct an exploratory numerical study with steady blowing applied to low-Reynolds-number flows around low-aspect-ratio wings. Steady forcing is considered here to restrict the parameter space (namely the actuation frequency). Similar studies to the current investigation are the circulation control ${ }^{24,25}$ that utilizes the Coanda effect at the trailing edge. The flow at the trailing edge can be redirected to increase the overall spanwise circulation of the wing and hence enhance lift. We emphasize that in the present investigation, our aim is not to prevent separation but is to exploit wake vortices by changing the threedimensional dynamics of the separated flow to enhance lift experienced by the low-aspect-ratio wings.

In the next section, we present the simulation methodology used to study the three-dimensional flows around the low-aspect-ratio rectangular flat-plate wings. Results for uncontrolled low-Reynolds-number separated flows are briefly presented with focus on the unsteady nature of vortex dynamics. Afterwards, the applications of steady blowing are considered to increase the lift exerted on the plate at post-stall angles of attack. Various actuator positions and locations are examined and two setups are identified for lift enhancement by utilizing tip effects. One of the effective control setups is further examined for a wide range of aspect ratios and angles of attacks and is found to increase lift in all cases.

\section{Simulation Method}

Three-dimensional incompressible flows around low-aspect-ratio wings are simulated with the immersed boundary projection method developed by Taira and Colonius. ${ }^{26}$ The flow field is discretized with a Cartesian grid and the wing is represented by a collection of Lagrangian boundary points where surface forces are applied to satisfy the no-slip condition. The continuous version of the governing equations for this method can be written in the following fashion:

$$
\begin{aligned}
\frac{\partial \mathbf{u}}{\partial t}+\mathbf{u} \cdot \nabla \mathbf{u} & =-\nabla p+\frac{1}{R e} \nabla^{2} \mathbf{u}+\int_{s} \mathbf{f}(\boldsymbol{\xi}(s, t)) \delta(\boldsymbol{\xi}-\mathbf{x}) d s \\
\nabla \cdot \mathbf{u} & =0 \\
\mathbf{u}(\boldsymbol{\xi}(s, t)) & =\int_{\mathbf{x}} \mathbf{u}(\mathbf{x}) \delta(\mathbf{x}-\boldsymbol{\xi}) d \mathbf{x}=\mathbf{u}_{B}(\boldsymbol{\xi}(s, t))
\end{aligned}
$$

where variables $\mathbf{u}, p$, and $\mathbf{f}$ are the non-dimensionalized velocity, pressure, and boundary force. All variables are non-dimensionalized appropriately with the chord length of the plate, $c$, the uniform flow velocity, $U_{\infty}$, and the kinematic viscosity of the fluid, $\nu$. The Reynolds number is defined as $R e \equiv U_{\infty} c / \nu$ and the spatial and temporal variables are non-dimensionalized as $x / c$ and $U_{\infty} t / c$, respectively.

Time integration is performed with the Crank-Nicolson and the second order Adam-Bashforth schemes for the viscous and the advective terms, respectively. The incompressibility constraint and the no-slip condition are satisfied through a single projection at each time level. The spatial domain is discretized with a second order finite-volume staggered grid formulation. Further details on the numerical method are described in Ref. 26.

The Courant number based on the free stream velocity has been set to $U_{\infty} \Delta t / \Delta x \leq 0.5$. This limit was found to be small enough to ensure sufficient temporal convergence and the results based on such time steps compared well with previous numerical and experimental studies for analogous two- and three-dimensional flows. ${ }^{13,26}$ In general, the accuracy of the current method is limited by the spatial discretization and not by the temporal discretization.

The rectangular wing is instantaneously materialized at $t=0^{+}$with its center at the origin in an initially uniform flow to replicate an impulsive translation. This initial condition is also equivalent to potential flow. A typical computational domain is $[-4,6.1] \times[-5,5] \times[-6,6]$ in the $x, y$, and $z$-directions (or streamwise, vertical, and spanwise-directions, respectively) with a grid size of $150 \times 66 \times 156$, which has been found sufficient from grid convergence studies performed previously. ${ }^{13}$ Grid stretching is applied to the spatial domain away from the body. The velocity boundary condition of uniform flow is specified at the computational boundary except for the outlet where the convective boundary condition, $\partial \mathbf{u} / \partial t+U_{\infty} \partial \mathbf{u} / \partial x=$ 0 , is applied. Validation of the computational setup is offered in Refs. 13 and 27, showing good agreement between the current simulation and the experiment for steady and unsteady flows around an impulsively started plate of $A R=2$ at $R e=100$. 
The forces on the rectangular wing of span $b$ and chord $c$ are reported in terms of the lift and drag coefficients defined by:

$$
C_{L}=\frac{F_{y}}{\frac{1}{2} \rho U_{\infty}^{2} b c} \quad \text { and } \quad C_{D}=\frac{F_{x}}{\frac{1}{2} \rho U_{\infty}^{2} b c}
$$

The wing is modeled to be infinitely thin in the simulations with a regularized body force dependent on the grid resolution. The regularization has not been observed to influence the flow field and the body force in any significant manner. ${ }^{27}$ The side force generated at high angles of attack in asymmetric flow (about the midspan) is found to be at least an order of magnitude smaller than the above two forces and is not focused upon in the current study. For discussions on the side forces, we refer readers to Ref. 13.

\section{Uncontrolled Flow}

We first consider the uncontrolled three-dimensional flow at a Reynolds number of 300. This Reynolds number is selected as the flows can exhibit separation and unsteadiness while remaining in the laminar regime. To illustrate the representative behavior of the flow around low-aspect-ratio wings, we select $A R=2$ in this section. The plate is impulsively translated at $t=0^{+}$, in an initially quiescent flow, with a fixed angle of attack to investigate the initial transient and long-time behavior of the wake vortices and the corresponding forces exerted on the plate.

Representative snapshots of the wake are shown in Fig. 1 for angles of attack of $\alpha=10^{\circ}, 20^{\circ}$, and $30^{\circ}$ with the corresponding lift and drag histories in Fig. 2. The wake vortices are visualized with two different iso-surfaces. The vorticity iso-surface is shown in light gray to capture the vortex sheets and the $Q$-criterion (or the $Q$-value), the second invariant of the velocity gradient tensor, is used to highlight the vortex cores depicted by the dark gray surfaces. Positive $Q$-values give prominence to regions of high swirl in comparison to shear to represent coherent vortices. ${ }^{28}$

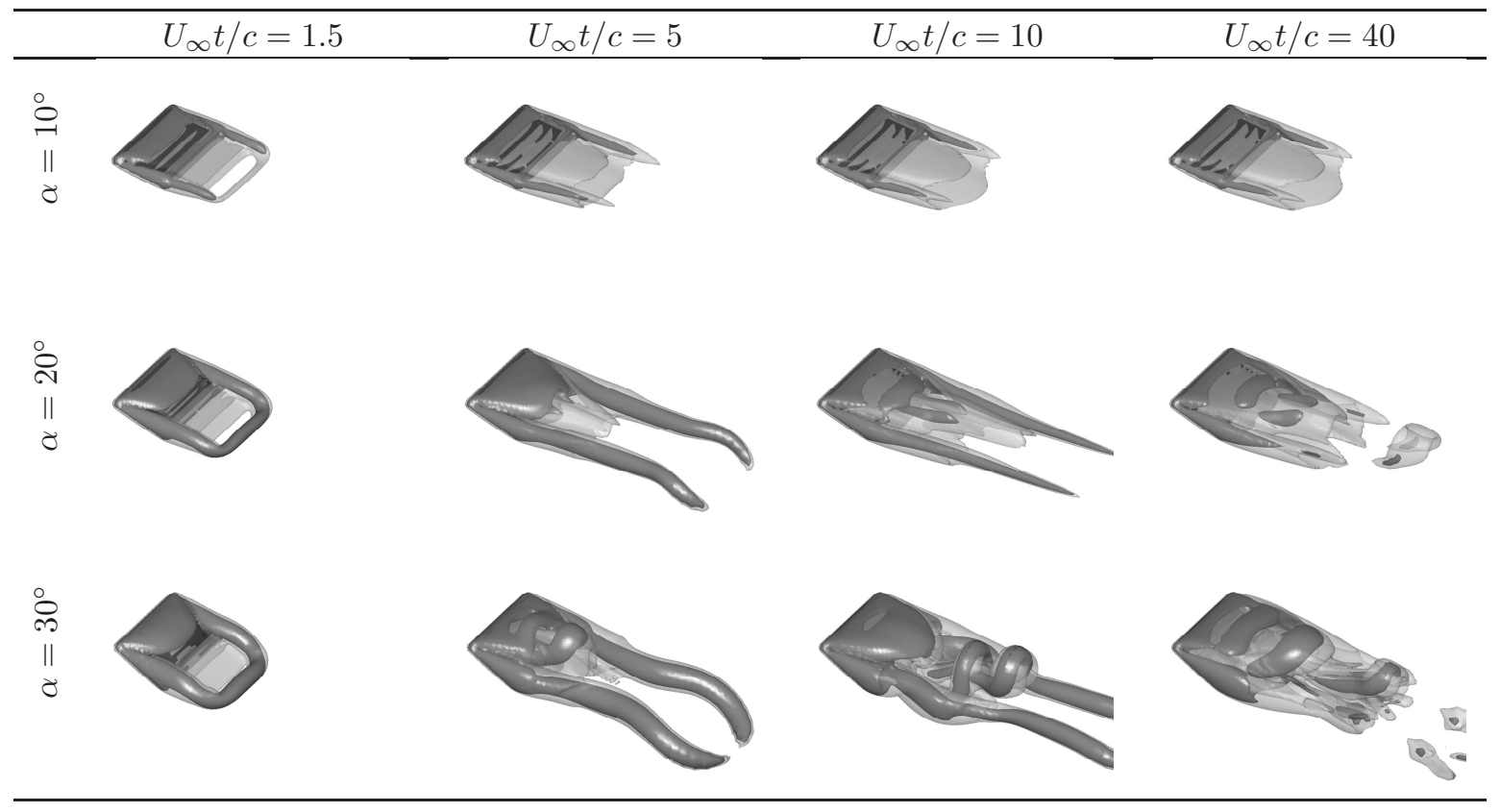

Figure 1. Top-port snapshots of the wake vortices behind rectangular plates of $A R=2$ at $\alpha=10^{\circ}$, $20^{\circ}$, and $30^{\circ}$. Shown are the iso-surfaces of $\|\boldsymbol{\omega}\|_{2}=2$ and $Q=1$.

Following the impulsive start, the topology of the wake vortices are similar for all angles of attack. The vortex sheets, generated by the initial acceleration and the edges of the plate, roll up and form the leadingedge, trailing-edge, and tip vortices (see Fig. 1 at $U_{\infty} t / c=1.5$ ). The low pressure core of the leading-edge vortex allows the plate to experience large lift (Fig. 2). The downward induced velocity from the tip vortices presses the leading-edge vortex onto the plate until about $t \approx 8$. Around this time the leading-edge vortex detaches and interacts with the tip vortices. The columnar structure of the tip vortices is then lost and 
the downward induced velocity applied to the leading-edge vortices is decreased. This process causes the consequent formation of leading-edge vortices to take place farther away from the top surface resulting in reduction of lift at large time (Fig. 2).

Later in time, the wake becomes stable and steady for low-aspect-ratio airfoils at small angles of attack. For example, the wake behind a plate of $A R=2$ at $\alpha=10^{\circ}$ reaches a steady state as shown in Fig. 1. As the angle of attack is increased, we observe unsteady shedding of the wake vortices at large time. At moderate angles of attack, the shedding is found to be periodic due to the detachment of the leading- and trailing-edge vortices. The wake at $\alpha=20^{\circ}$ exhibits such periodic behavior in Fig. 1. For much higher angles of attack, the tip vortices interact strongly with the leading- and trailing-edge vortices resulting in aperiodic wake vortices over time (see $\alpha=30^{\circ}$ in Fig. 1), and are also found to be asymmetric about the midspan. This asymmetry has also been observed by Jian and Ke-Qin ${ }^{29}$ in flows around low-aspect-ratio elliptic wings at high angles of attack.
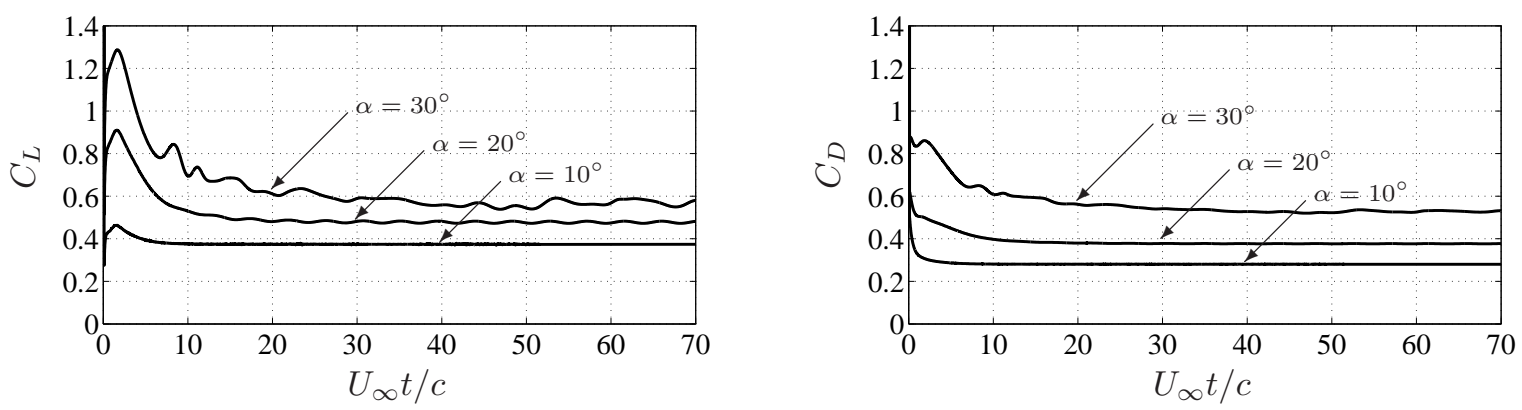

Figure 2. Lift and drag on a rectangular plate of $A R=2$ for unactuated flows of $\operatorname{Re}=300$ at $\alpha=10^{\circ}, 20^{\circ}$, and $30^{\circ}$.

Flows around other aspect ratios and planform shapes were investigated experimentally by Torres and Mueller $^{11}$ at $R e \approx 10^{5}$ and numerically by Taira and Colonius ${ }^{13}$ at $R e=300$ and 500 . The two most important parameter influencing the dynamics of the wake vortices are the aspect ratio and the angle of attack of the plate, which will be considered in the context of controlled flow below. A stability diagram for the large-time behavior of the uncontrolled wake is presented in Ref. 13 over a parameter space of angle of attack and aspect ratio.

\section{Controlled Flow}

\section{Actuator model}

In the following simulations, we introduce a body force to model steady blowing. This time-invariant force is applied to the flow field as a uniform strip along the span expressed as

$$
\mathbf{f}_{\mathrm{act}}=\hat{\mathbf{f}}_{\mathrm{act}} \delta\left(x-x_{0}\right) \delta\left(y-y_{0}\right) \Gamma\left(-z+\frac{b}{2}\right) \Gamma\left(z+\frac{b}{2}\right)
$$

and is added to the right-hand side of the momentum equation, Eq. (1). For the current model, addition of mass to the system is not taken into account. Here $\hat{\mathbf{f}}_{\text {act }}$ prescribes the strength and the direction of the actuator. The location of the actuator is specified with $\left(x_{0}, y_{0}\right)$ in the spanwise plane and $b$ denotes the span of the plate. The function $\Gamma($ ) corresponds to the Heaviside step function representing a strip in the spanwise direction. In the computations, the Dirac delta function, $\delta()$, is replaced by a discrete delta function, $\tilde{\delta}()$, proposed by Roma et al. ${ }^{30}$ that regularizes the singularity across 3 cells in both the $x$ - and $y$-directions in the following manner:

$$
\tilde{\delta}(x)= \begin{cases}\frac{1}{6 \Delta x}\left[5-3 \frac{|x|}{\Delta x}-\sqrt{-3\left(1-\frac{|x|}{\Delta x}\right)^{2}+1}\right] & \text { for } 0.5 \Delta x \leq|x| \leq 1.5 \Delta x, \\ \frac{1}{3 \Delta x}\left[1+\sqrt{-3\left(\frac{x}{\Delta x}\right)^{2}+1}\right] & \text { for }|x| \leq 0.5 \Delta x \\ 0 & \text { otherwise. }\end{cases}
$$


The function is shown here for the $x$-direction with a mesh width of $\Delta x$. This delta function is also used in the immersed boundary projection method to represent the immersed boundary.

The discrete delta function is selected to use the smallest possible width for the actuator, which is limited by the resolution of the simulation. For the current actuator model, there is not a direct analog for the physical slot width, since the simulated blowing does not exit through a slot. Hence, the modeled slot width is set to the characteristic length scale of the discrete delta function, $\sigma=\Delta x=0.04 c$. Compared to the typical slot widths of $\sigma / c \approx 0.01$ used in flow control, ${ }^{31-33}$ the current slot width in our simulations is slightly larger. Nonetheless the present forcing function is used to explore control techniques at this low Reynolds number.

The actuator is not placed exactly on the surface of the plate to avoid the numerical interference of the boundary force used in the immersed boundary method. The controller is positioned $3 \Delta x$ from the plate on the suction side. Forcing directions of upstream, sideways (outward from the midspan to the wing tip), and downstream are considered. In the present study, forcing directions are always set to be tangential to the wing surface. A representative flow control setup is provided in Fig. 3.

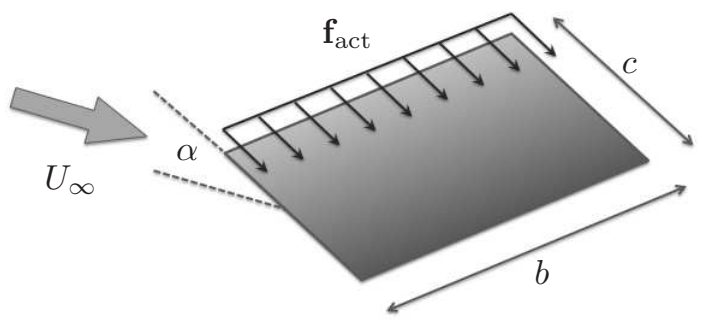

Figure 3. A flow control setup shown for an example of actuation along the leading edge in the downstream direction.

\section{Strength of actuation}

The strength of the actuation is reported in the present study with the non-dimensional momentum coefficient:

$$
C_{\mu} \equiv \frac{\rho U_{\mathrm{act}}^{2} b \sigma_{\mathrm{act}}}{\frac{1}{2} \rho U_{\infty}^{2} b c}=2\left(\frac{U_{\mathrm{act}}}{U_{\infty}}\right)^{2}\left(\frac{\sigma_{\mathrm{act}}}{c}\right)
$$

where $U_{\text {act }}$ is the actuator velocity. To characterize the actuator model, we simulate this steady blowing with a prescribed $\hat{\mathbf{f}}_{\mathrm{act}}$ in an initially quiescent free space. Once steady state is achieved, the velocity at the center of forcing is selected as the characteristic velocity $U_{\text {act }}$. For example, $\left|\hat{\mathbf{f}}_{\text {act }}\right|=0.1$ corresponds to $U_{\text {act }} / U_{\infty}=0.356$ and $C_{\mu}=1.0 \%$.

Next, we consider the strength of actuation required to alter the flow field in a noticeable manner. In order to alter the dynamics of the wake vortices in a low-Reynolds-number flow, rather large values of $C_{\mu}$ are selected to overcome the viscous effect (and due to the large slot width limited by the grid resolution). Standard values of the momentum coefficient in past studies have ranged from $C_{\mu}=0.01 \%$ to $10 \%$ for applications of steady blowing on wings. ${ }^{31-33}$ To illustrate the change in the forces exerted upon the plate, we apply flow control around a rectangular plate of $A R=2$ at $\alpha=30^{\circ}$ and $R e=300$. This example is chosen as the wing is at very high post-stall angle of attack generating strong interaction of the wake vortices and asymmetry about the midspan in the unactuated case. ${ }^{13}$ Actuation along the leading edge in the downstream direction with $C_{\mu}=0.10 \%, 0.51 \%$, and $1.0 \%$ are considered.

Figure 4 exhibits the changes in the lift and drag forces resulting from the leading-edge actuation with varying $C_{\mu}$. With an actuation effort of $C_{\mu}=0.10 \%$, there are no pronounced changes in the forces. As the momentum coefficient is increased to $0.51 \%$, lift starts to show increase from the unactuated case. It is interesting to note that the drag history is less affected by the actuation in comparison to the lift increase. With $C_{\mu}=1.0 \%$, a significant increase in lift of $33.8 \%$ is observed at large time in a timeaverage sense. Steady blowing here is modeled through a body force with a magnitude in the lift direction of $\int\left|\mathbf{f}_{\text {act }}\right| d V \sin 30^{\circ} / \frac{1}{2} \rho U_{\infty}^{2} b c=0.1$. Lift enhancement beyond this value can be attributed to the vortical forces. In what follows we consider the use of $C_{\mu}=1.0 \%$ to explore actuation locations and directions for the 

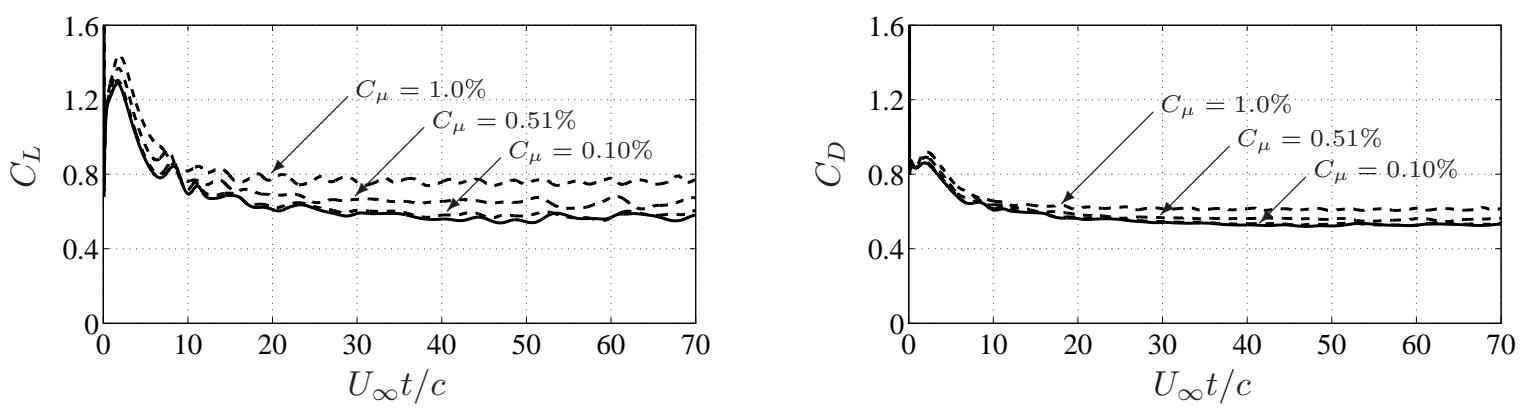

Figure 4. Forces on the plate with leading-edge actuation for $C_{\mu}=0.10 \%, 0.51 \%$, and $1.0 \%$. Solid and dash lines correspond to unactuated and actuated cases, respectively.

same example problem. Once a favorable setup for flow control is identified, other conditions are examined later in this paper.

\section{Location and direction of actuation}

Below we consider the application of steady blowing along the leading edge, midchord, and trailing edge in the upstream, sideways, and downstream directions with $C_{\mu}=1.0 \%$. Here, forcing directions are taken to be parallel to the surface of the plate for all cases. For example, downward actuation would be applied with $\hat{\mathbf{f}}_{\mathrm{act}}=\hat{\mathrm{f}}_{\mathrm{act}}\left(\cos \alpha \hat{\mathbf{e}}_{x}-\sin \alpha \hat{\mathbf{e}}_{y}\right)$. Sideways actuation is directed outward from the midspan to the tips of the wing. A collection of lift and drag histories from the controlled flows are presented in Fig. 5.

The top two plots in Fig. 5 show the force histories for the cases of leading-edge actuation. Lift is increased with downstream blowing, as the separated flow structures become closer to the surface of the plate. The corresponding low-pressure vortex cores sit directly above the top surface enhancing lift by $34 \%$ as mentioned in the previous section. The downstream blowing also repositions the wake vortices downward past the trailing edge increasing the effective frontal area. This in turn causes the drag to increase by $16 \%$, a smaller amount compared to lift.

With flow control in the upstream direction, the leading-edge vortices are pushed into the freestream from the top surface, resulting in the loss of the low-pressure region near the surface and, accordingly, a decrease in lift. Outward forcing was applied in hope of releasing the vorticity generated by the leading edge by inducing spanwise flow from the midspan to the tips ${ }^{\mathrm{a}}$. However, the right-angled corners on the wing separate the vortex sheets emanating from the leading edge and tip, and triggered the roll up of individual structures (i.e., the leading-edge and tip vortices). Hence the leading-edge vortex and the tip vortices remained disconnected at all times without merging or connecting, in spite of sideways blowing. The overall wake structure was wider in the spanwise direction than the unactuated flow but did not have much influence on the lift or drag exerted on the plate.

The middle two plots in Fig. 5 illustrate the effect of midchord actuation onto the lift and drag histories. The noticeable change from the control comes from the downstream forcing where the flow achieves stable steady state and a $76 \%$ increase in lift by creating an elongated wake structure (shown in the next section). The vorticity produced by the plate is stably released to the freestream from the leading-edge vortex sheet and the tip vortices at this low Reynolds number. Although this actuator setup is attractive, it would be unlikely to yield a steady flow at higher Reynolds numbers since maintaining such stable wake structure is highly dependent on viscous diffusion. We note that the drag is also affected as the wake structure is moved downward with downstream blowing.

Another change in the dynamics of the wake is exhibited by the sideways blowing along the midchord. This control arrangement repositions the tip vortices away from the plate and allows the leading- and trailingedge vortices to roll up and shed in a periodic manner. Such behavior of the flow results in a large fluctuation of lift on the wing.

Out of the locations considered in Fig. 5, the wake is found to be most sensitive to momentum injection at the trailing edge. The lift shows significant increase and decrease with the application of downstream

\footnotetext{
${ }^{a}$ Spanwise blowing has been shown by Campbell ${ }^{34}$ to generate large increase in lift at high angles of attack for swept wings with $C_{\mu}=4 \%$ to $31 \%$.
} 

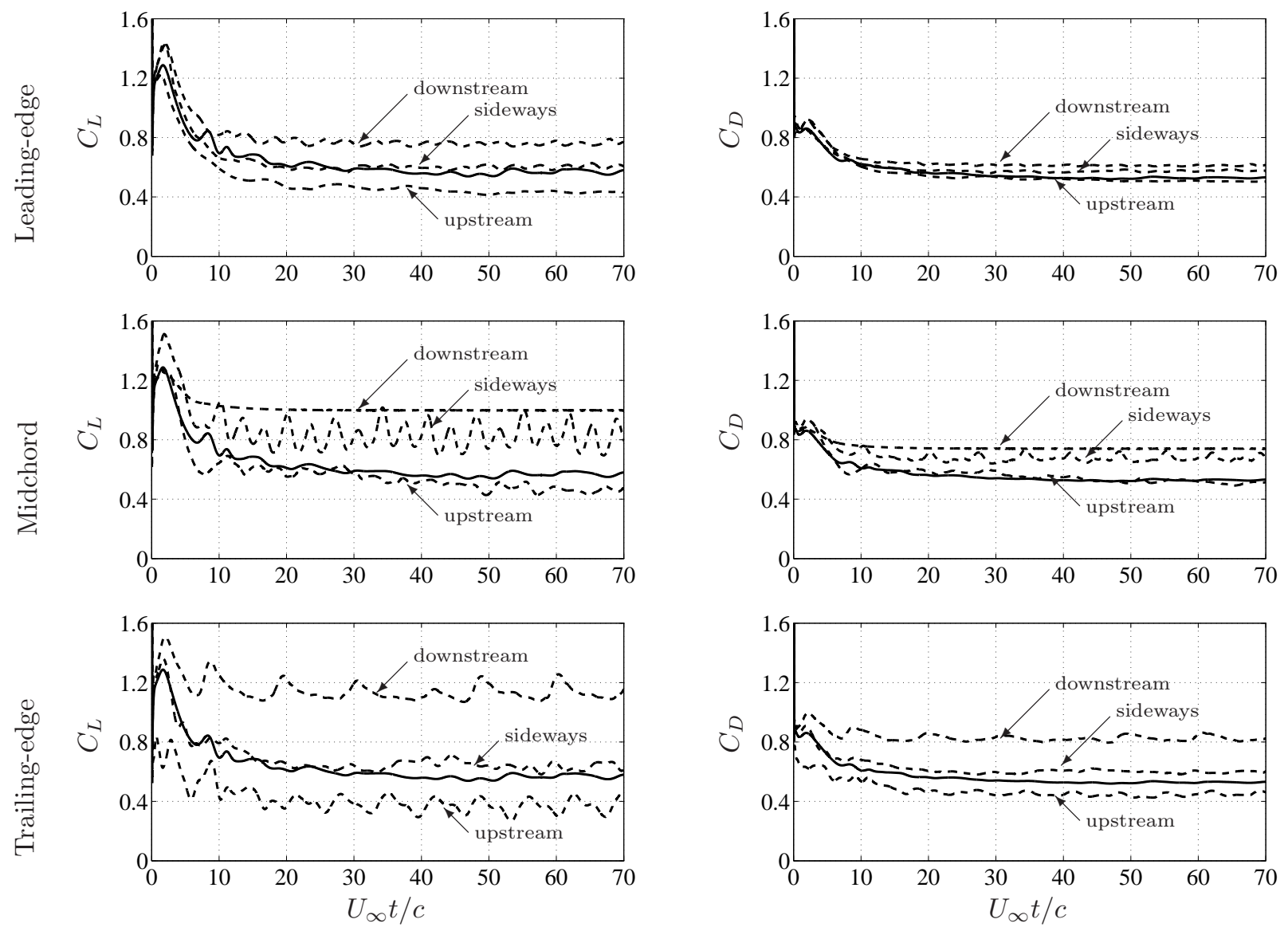

Figure 5. Lift and drag histories for cases of leading-edge, midchord, and trailing-edge actuation with $C_{\mu}=1.0 \%$ applied to the separated flow around a rectangular plate of $A R=2$ at $\alpha=30^{\circ}$ and $R e=300$. Solid and dash lines correspond to unactuated and actuated cases, respectively. 
and upstream forcing, respectively. The time-average lift is increased by a remarkable $100 \%$ for downstream blowing. Such noticeable changes in forces are not realized for the sideways blowing at the trailing edge.

The reason for the strong influence of the actuation upon the forces is the direct modification of the trailing-edge vortex. Upstream and downstream blowing, respectively, encourages and discourages the interaction between the leading- and trailing-edge vortices. Below, we will further examine how the downstream actuation at the trailing edge modifies the vortex dynamics in the vicinity of the wing and contributes to lift enhancement.

To summarize, we have observed that steady blowing can change the dynamics of the wake vortices to increase or decrease lift and drag. From the three locations considered, the trailing edge is observed to affect the force on the wing in the most substantial manner. At all three actuator positions, steady blowing in the downstream direction enhanced lift whereas the upstream direction reduced lift. The most effective actuator for the considered example is found to be at the trailing edge in the downstream direction, doubling in lift at large time from the change in the dynamics of the wake vortices.

\section{Wake modification with actuation}

Let us visualize the flow field around the rectangular plate of $A R=2$ at $\alpha=30^{\circ}$ and $R e=300$ for the two most effective cases of actuation from the above discussion, namely the downstream blowing at the midchord and at the trailing edge. Similar to the flow visualization employed in Fig. 1, representative snapshots of the wake vortices at large time $\left(U_{\infty} t / c=70\right)$ with and without actuation are illustrated in Fig. 6 with the iso-surfaces of $\|\boldsymbol{\omega}\|_{2}=2$ and $Q=1$. Also presented are the time-average pressure fields and the streamlines along the midspan.

(a) no control
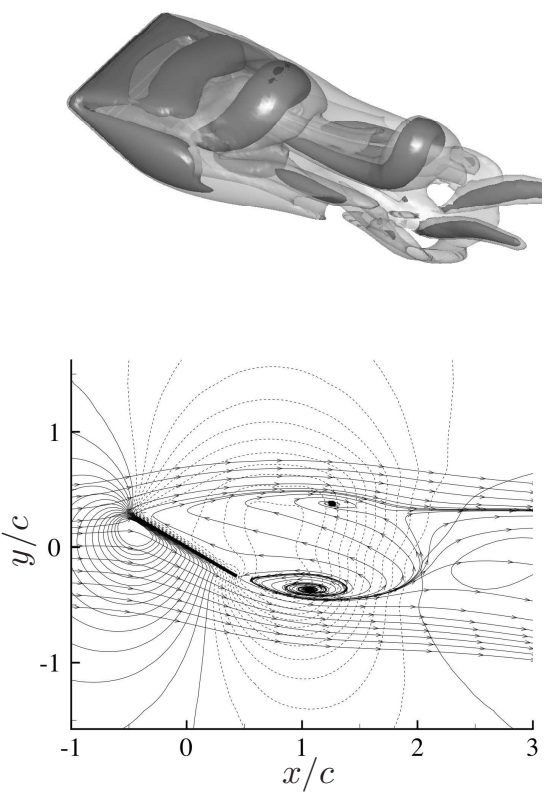

(b) midchord actuation
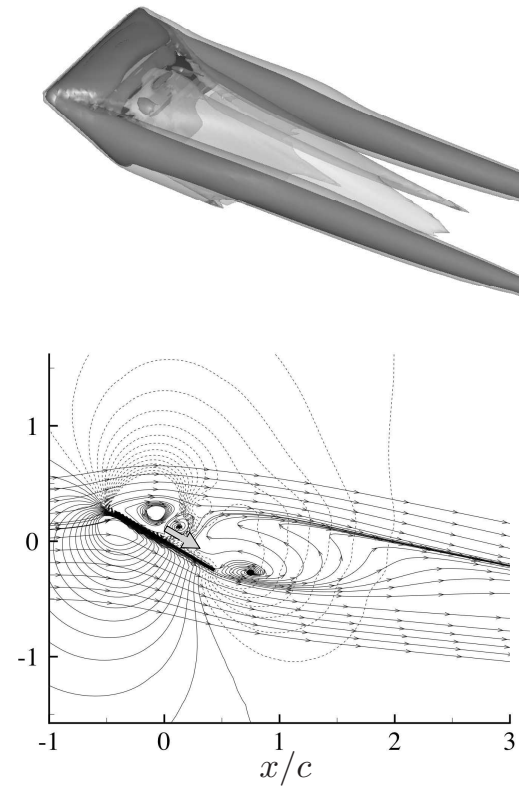

(c) trailing-edge actuation
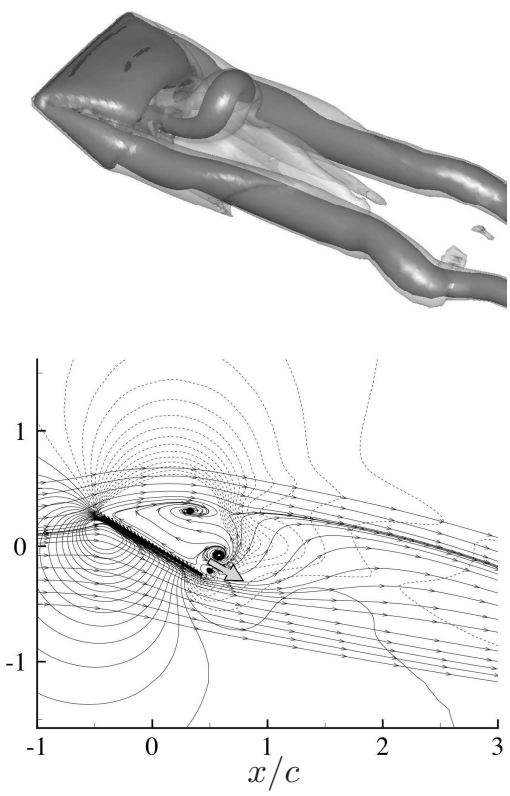

Figure 6. (Top) Snapshots of the large-time wake around a rectangular wing of $A R=2$ at $\alpha=30^{\circ}$ and $R e=300$. (Bottom) Corresponding time-average pressure distribution and streamlines along the midspan. Pressure contour levels are set from -0.3 to 0.3 in increments of 0.04 with the negative pressure shown by the dashed lines. Arrows indicate the location of actuation.

The unactuated flow exhibits complex interaction amongst the leading-edge, trailing-edge, and tip vortices with the dominant flow structures mostly consisting of the leading- and trailing-edge vortices. For the two controlled cases, we observe that the most dominant flow features are the long columnar tip vortices formed by engulfing the vortex sheet from the trailing edge. The downstream blowing along the midchord allows for the flow around the wing to achieve a steady state as shown with its elongated wake vortices in Fig. 6(b). The tip vortices exhibit clear columnar structures generating downward induced velocity onto the wake stabilizing 
the leading-edge vortex sheet. The vorticity within this sheet is diffused into the free stream in a steadfast manner at this Reynolds number. In the case of downstream blowing at the trailing edge, the strengthened tip vortices apply stronger downward induced velocity on the leading-edge vortices and let them roll up in close neighborhood of the top surface of the plate as shown in Fig. 6(c). Hence the low-pressure cores from the roll-up provide lift enhancement.

In both cases the separation bubble with control visualized along the midspan have become smaller in a time-average sense compared to the unactuated case. Additionally, the streamlines are deflected further downward with blowing, directly implying that lift on the wing is increased based on the momentum balance. Note that the roll-up motion at the trailing edge from the unactuated case is now attenuated with steady blowing. The removal of such roll-up seems to be the key factor in modifying the dynamics of the wake.

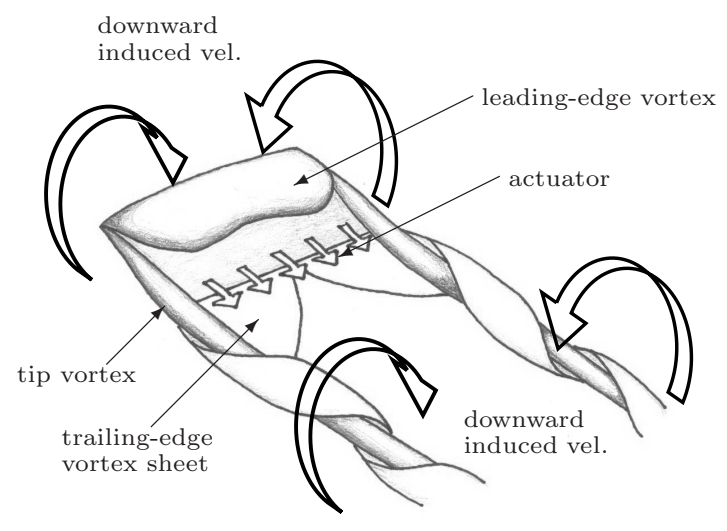

Figure 7. An illustration of tip vortices engulfing the trailing-edge vortex sheet with trailing-edge actuation.

To illustrate the lift enhancement mechanism, we present Fig. 7 for the trailing-edge blowing but this figure also captures the flow physics for the case of midchord blowing. With downstream blowing, the trailing-edge vortex sheet is pushed further downward avoiding direct interaction with the vortical structure emanating from the leading edge. As the trailing-edge vortex sheet advects downstream, it is rolled into the tip vortices which in turn strengthens the tip vortices. Consequently, the strong downward velocity induced by the tip vortices presses the leading-edge vortex and the corresponding low-pressure core region onto the top surface of the wing, enhancing lift. Hence, we suggest that the tip vortices can be used as effectively as the leading-edge vortices in applying vortical forces upon a body. While most of the past discussions in circulation control ${ }^{24}$ and flapping-wing aerodynamics ${ }^{17}$ have focused on the spanwise circulation to explain the lift enhancements, streamwise circulation (tip vortices) can also contribute to lift with the current control setup. Traditionally, the tip effects are thought of as a nuisance due to the loss of the low-pressure region near the tips for attached flows at small angles of attack. However, we emphasize here that the tip vortices can be used favorably to enhance lift in separated flows behind low-aspect-ratio wings.

\section{Parametric study for downstream blowing at the trailing edge}

For the most effective case of downstream blowing at the trailing edge, we now further examine the effect of aspect ratio, momentum coefficient, and angle of attack.

The time-average lift and lift-to-drag ratio at large time are summarized in Fig. 8. We observe increase in lift and lift-to-drag ratio over a range of angle of attack for $C_{\mu}=0.51 \%$ and $1.0 \%$. Shown on the left are the time-average lift coefficients with actuation as well as the time-average and maximum lift coefficients without actuation. What is denoted as the maximum lift is achieved immediately following the impulsive start for the uncontrolled cases around $U_{\infty} t / c \approx 1.7$ (Fig. 1). The difference between the average and maximum lift for the uncontrolled flow is attributed to the lift enhancement provided by the existence of the initial leading-edge vortex. Enhanced lift from steady blowing is now close to or larger than the maximum lift achieved immediately after the impulsive start, as well as the inviscid limit of lift for low-aspect-ratio 
airfoils ${ }^{\mathrm{b}}$.

Let us examine the performance of the actuator for the wing of $A R=1$. In Fig. 8, we observe that the time-average lift with control is increased past the maximum lift achieved by the uncontrolled transient flow for almost all cases considered. Again, this increase is not from the addition of the numerical body force used to represent steady blowing. The direct contributions to lift from such modeled blowing are $0.1 \sin \alpha$ and $0.2 \sin \alpha$ for $C_{\mu}=0.51 \%$ and $1.0 \%$, respectively, and do not account for the full increase realized here. The lift increase seen here results mostly from the modification of the wake vortices.

With flow control around a wing of $A R=2$, the time-average lift is further increased especially around $\alpha \approx 20^{\circ}$. For higher $\alpha$, the interaction of the wake vortices reduce the level of increase but still achieves an overall enhancement for both forcing magnitudes. A similar trend holds for the case of $A R=4$ with peak performance around $\alpha \approx 20^{\circ}$. In the case of $A R=1$, the tip vortices cover almost the entire span without leaving much room for the leading-edge vortex to stay near the top surface. The lift-to-drag ratio does not show a large enhancement for $A R=1$ when compared to the higher-aspect-ratio wings. In the case of larger aspect-ratio wings, we observe large increase in lift due to the large extent of the leading-edge vortex over the span.

Once the flow becomes overwhelmed with strong nonlinear interaction of the wake vortices at high angles of attack (i.e., $\alpha \approx 40^{\circ}$ ), the amount of lift enhancement is reduced. In such high-angle-of-attack flows, downstream blowing is not able to keep the trailing-edge vortex sheet from interacting with other vortices and results in no significant increase in the strength of the tip vortices (unless perhaps with much stronger blowing). Nonetheless, this actuator setup seems to be effective overall for various regimes (steady and unsteady periodic/aperiodic states as discussed in the uncontrolled flow section). The strip of steady blowing at the trailing edge is especially attractive for $A R \gtrsim 2$ and $\alpha \lesssim 30^{\circ}$ as the lift-to-drag ratio shows substantial increase as well.

To demonstrate that the increase in lift is attributed to the strengthening of the tip vortices due to the downstream trailing-edge actuation, we compute the relative increase in time-average (avg) lift and streamwise circulation of the tip vortex:

$$
R_{L} \equiv \operatorname{avg} C_{L} / \operatorname{avg} C_{L}^{*} \quad \text { and } \quad R_{\Gamma} \equiv \operatorname{avg} \Gamma / \operatorname{avg} \Gamma^{*},
$$

respectively, where $*$ is used here to denote the unactuated results. The circulation of the tip vortex is evaluated at a streamwise location of $x / c=2.5$ using $\Gamma=\oint \mathbf{u} \cdot d \mathbf{l}$, where the contour is chosen to enclose the patch of vorticity (tip vortex) above $1 \%$ of the maximum value. These relative increases are plotted against each other in Fig. 9 for actuated cases with $C_{\mu}=0.51 \%$ and $1.0 \%$ around wings of $A R=1,2$, and 4. Based on Fig. 9, the correlation coefficient $\rho\left(R_{\Gamma}, R_{L}\right)$ is found to be 0.952 , which indeed suggests that strengthening the tip vortices have positive influence on the lift enhancement for low-aspect-ratio wings at post-stall angles of attack. With the current flow control arrangement, an increase in lift as high as about 2.5 times the unactuated value has been achieved for one case.

\section{Conclusion}

We considered the application of steady blowing to separated flows behind low-aspect-ratio rectangular wings at a low Reynolds number. The objective of the flow control was to enhance lift at post-stall angles of attack by changing the three-dimensional dynamics of the wake vortices. Flow control was simulated with the immersed boundary projection method and the actuator was modeled by a body force near the top surface of the wing to represent steady tangential blowing. Various setups were considered and downstream blowing along the trailing edge was found to be most effective in enhancing lift. This controller strengthened the tip vortices by engulfing the trailing-edge vortex sheet to increase the downward induced velocity onto the leading-edge vortices. The low-pressure cores of the leading-edge vortices moved closer to the top surface contributing to the increase in lift. In some ways, the present control mechanism is similar to circulation control. ${ }^{24}$ However, instead of simply increasing the spanwise circulation, we have increased the streamwise circulation of the tip vortices here. The tip vortices that are traditionally considered as an aerodynamic nuisance, have been used in favor to increase lift in post-stall flows for the considered low-aspect-ratio wings.

\footnotetext{
${ }^{\mathrm{b}}$ The three-dimensional inviscid lift limit, $C_{L}=2 \pi \alpha /\left(\sqrt{1+(2 / A R)^{2}}+2 / A R\right)$, was derived by Helmbold ${ }^{35}$ from the lifting surface theory for elliptic wings and is shown to be in remarkable agreement with low-aspect-ratio wings of $A R<4$. Lift for rectangular planforms of $0.5 \leq A R \leq 6$ is accurately predicted with this model. ${ }^{36}$
} 

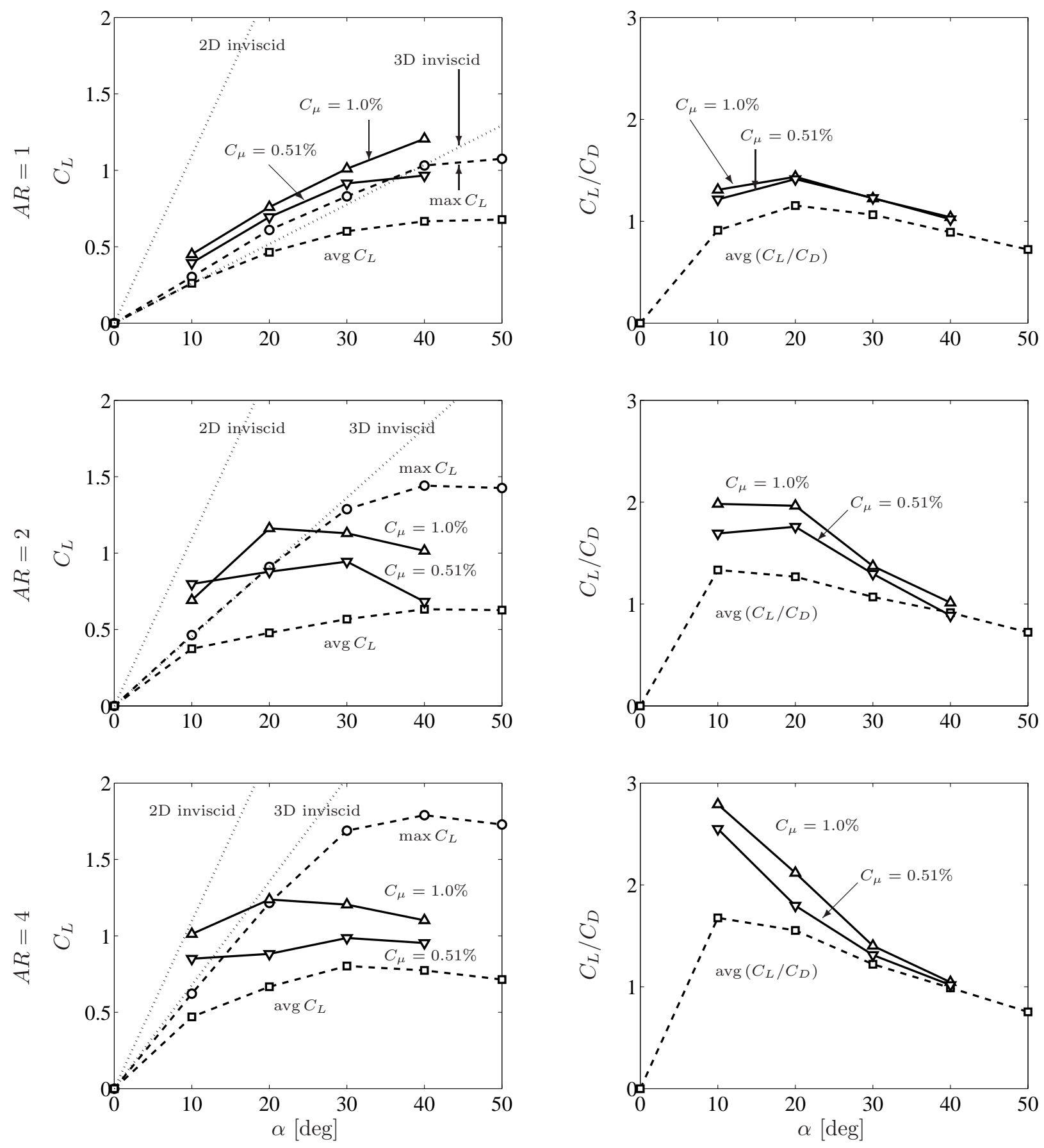

Figure 8. Time-average lift coefficients and lift-to-drag ratios for wings of $A R=1,2$, and 4 for cases without actuation $(\square)$ and with actuation for $C_{\mu}=0.51 \%(\nabla)$ and $1.0 \%(\triangle)$. Shown also are the maximum lift for unactuated case $(\circ)$ and the inviscid limits ( ………...... $)$. 


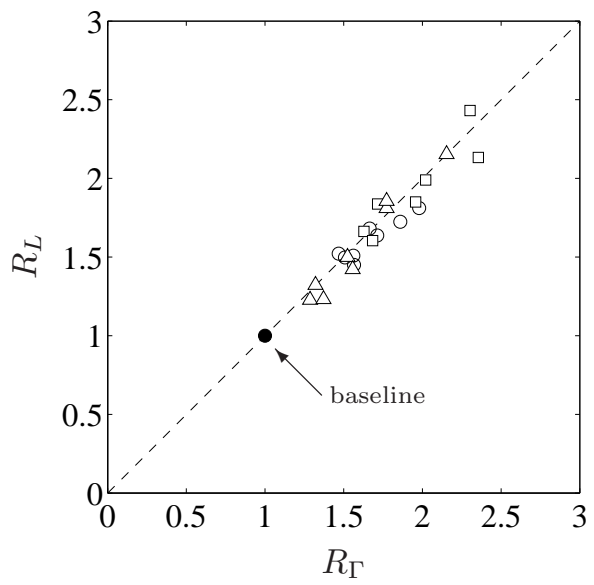

Figure 9. Normalized lift over normalized circulation of the tip vortex from control $(A R=1: \square, A R=2: \circ$, and $A R=4: \triangle)$.

Concurrent research is underway to investigate active control of low-Reynolds-number separated flows over two- and three-dimensional (low-aspect-ratio) wings with feedback mechanism ${ }^{37}$ as well as adjoint analysis. ${ }^{38}$

\section{Acknowledgments}

This work was supported by the US Air Force Office of Scientific Research (FA9550-05-1-0369) with some of the computations made possible by the US Department of Defense High Performance Computing Modernization Program. We are thankful to Professors Clarence W. Rowley, Morteza Gharib, David R. Williams, Gilead Tadmor, and Dr. William B. Dickson for the enlightening discussions.

\section{References}

${ }^{1}$ Grasmeyer, J. and Keenon, M., "Development of the Black Widow micro air vehicle," 2001, AIAA Paper 2001-012\%.

${ }^{2}$ Ho, S., Nassef, H., Pornsinsirirak, N., Tai, Y.-C., and Ho, C.-M., "Unsteady aerodynamics and flow control for flapping wing flyers," Progress in Aerospace Sciences, Vol. 39, 2003, pp. 635-681.

${ }^{3}$ Bohorquez, F., Samuel, P., Sirohi, J., Pines, D., Rudd, L., and Perel, R., "Design analysis and hover performance of a rotary wing micro air vehicle," Journal of the American Helicopter Society, Vol. 48(2), 2003, pp. 80-90.

${ }^{4}$ Stanford, B., Ifju, P., Albertani, R., and Shyy, W., "Fixed membrane wings for micro air vehicles: experimental characterization, numerical modeling, and tailoring," Progress in Aerospace Sciences, Vol. 44, 2008, pp. 258-294.

${ }^{5}$ Mueller, T. J., editor, Fixed and flapping wing aerodynamics for micro air vehicle applications, Vol. 195 of Progress in Astronautics and Aeronautics, AIAA, 2001.

${ }^{6}$ Mueller, T. J. and DeLaurier, J. D., "Aerodynamics of small vehicles," Annual Review of Fluid Mechanics, Vol. 35, 2003, pp. 89-111.

${ }^{7}$ Pines, D. J. and Bohorquez, F., "Challenges facing future micro-air-vehicle development," Journal of Aircraft, Vol. 34(2), 2006, pp. 290-305.

${ }^{8}$ Freymuth, P., Finaish, F., and Bank, W., "Further visualization of combined wing tip and starting vortex systems," AIAA Journal, Vol. 25(9), 1987, pp. 1153-1159.

${ }^{9}$ Pelletier, A. and Mueller, T. J., "Low Reynolds number aerodynamics of low-aspect-ratio, thin/flat/cambered-plate wings," Journal of Aircraft, Vol. 37(5), 2000, pp. 825-832.

${ }^{10}$ Sunada, S., Yasuda, T., Yasuda, K., and Kawachi, K., "Comparison of wing characteristics at an ultralow Reynolds number," Journal of Aircraft, Vol. 39(2), 2002, pp. 331-338.

${ }^{11}$ Torres, G. E. and Mueller, T. J., "Low-aspect-ratio wing aerodynamics at low Reynolds numbers," AIAA Journal, Vol. 42(5), 2004, pp. 865-873.

${ }^{12}$ Cosyn, P. and Vierendeels, J., "Numerical investigation of low-aspect-ratio wings at low Reynolds numbers," Journal of Aircraft, Vol. 43(3), 2006, pp. 713-722.

${ }^{13}$ Taira, K. and Colonius, T., "Three-dimensional separated flows around low-aspect-ratio flat plates," Journal of Fluid Mechanics, 2008, accepted.

${ }^{14}$ Dickinson, M. H. and Götz, K. G., "Unsteady aerodynamic performance of model wings at low Reynolds numbers," Journal of Experimental Biology, Vol. 174, 1993, pp. 45-64. 
${ }^{15}$ Ellington, C. P., van den Berg, C., Willmott, A. P., and Thomas, A. L. R., "Leading-edge vortices in insect flight," Nature, Vol. 384, 1996, pp. 626-630.

${ }^{16}$ Birch, J. M. and Dickinson, M. H., "Spanwise flow and the attachment of the leading-edge vortex on insect wings," Nature, Vol. 412, 2001, pp. 729-733.

${ }^{17}$ Birch, J. M., Dickson, W. B., and Dickinson, M. H., "Force production and flow structure of the leading edge vortex on flapping wings at high and low Reynolds numbers," Journal of Experimental Biology, Vol. 207, 2004, pp. $1063-1072$.

${ }^{18}$ Poelma, C., Dickson, W. B., and Dickinson, M. H., "Time-resolved reconstruction of the full velocity field around a dynamically-scaled flapping wing," Experiments in Fluids, Vol. 41, 2006, pp. 213-225.

${ }^{19}$ Choi, H., Jeon, W.-P., and Kim, J., "Control of Flow Over a Bluff Body," Annual Review of Fluid Mechanics, Vol. 40, 2008, pp. 113-139.

${ }^{20}$ Watkins, S., Milbank, J., Loxton, B. J., and Melbourne, W. H., "Atmospheric winds and their implications for microair vehicles," AIAA Journal, Vol. 44(11), 2006, pp. 2591-2600.

${ }^{21}$ Seifert, A., Darabi, A., and Wygnanski, I., "Delay of airfoil stall by periodic excitation," Journal of Aircraft, Vol. 33(4), 1996, pp. 691-698.

${ }^{22}$ Greenblatt, D., Neuburger, D., and Wygnanski, I., "Dynamic stall control by intermittent periodic excitation," Journal of Aircraft, Vol. 38(1), 2001, pp. 188-190.

${ }^{23}$ Seifert, A., Greenblatt, D., and Wygnanski, I. J., "Active separation control: an overview of Reynolds and Mach numbers effects," Aerospace Science and Technology, Vol. 8, 2004, pp. 569-582.

${ }^{24}$ Englar, R. J., "Circulation control pneumatic aerodynamics: blown force and moment augmentation and modification; past, present \& future," 2000, AIAA Paper 2000-2541.

${ }^{25}$ Joslin, R. D. and Jones, G. S., editors, Applications of Circulation Control Technologies, Vol. 214 of Progress in Astronautics and Aeronautics, AIAA, 2006.

${ }^{26}$ Taira, K. and Colonius, T., "The immersed boundary method: a projection approach," Journal of Computational Physics, Vol. 225, 2007, pp. 2118-2137.

${ }^{27}$ Taira, K., Dickson, W. B., Colonius, T., Dickinson, M. H., and Rowley, C. W., "Unsteadiness in flow over a flat plate at angle-of-attack at low Reynolds numbers," 2007, AIAA Paper 2007-710.

${ }^{28}$ Hunt, J. C. R., Wray, A. A., and Moin, P., "Eddies, stream, and convergence zones in turbulent flows," Tech. Rep. CTR-S88, Center for Turbulence Research, 1988.

${ }^{29}$ Jian, T. and Ke-Qin, Z., "Numerical and experimental study of flow structure of low-aspect-ratio wing," Journal of Aircraft, Vol. 41(5), 2004, pp. 1196-1201.

${ }^{30}$ Roma, A. M., Peskin, C. S., and Berger, M. J., "An adaptive version of the immersed boundary method," Journal of Computational Physics, Vol. 153, 1999, pp. 509-534.

${ }^{31}$ Lee, C. S., Tavella, D., Woods, N. J., and Roberts, L., "Flow structure and scaling laws in lateral wing-tip blowing," AIAA Journal, Vol. 27(8), 1989, pp. 1002-1007.

${ }^{32}$ Duraisamy, K. and Baeder, J. D., "Numerical simulation of the effects of spanwise blowing on tip vortex formation," Journal of Aircraft, Vol. 43(4), 2006, pp. 996-1006.

${ }^{33}$ Holloway, A. G. L. and Richardson, S., "Development of a trailing vortex formed with spanwise tip jets," Journal of Aircraft, Vol. 44(3), 2007, pp. 845-857.

${ }^{34}$ Campbell, J. F., "Augmentation of vortex lift by spanwise blowing," Journal of Aircraft, Vol. 13(9), 1976, pp. 727-732.

${ }^{35}$ Helmbold, H. B., "Der unverwundene ellipsenflugel als tragende flanche," Tech. rep., Jahrbuch 1942 der Deutch Luftfahrtforsch, 1942

${ }^{36}$ Anderson, J. D., Aircraft performance and design, McGraw-Hill, Boston, 1999.

${ }^{37}$ Joe, W. T., Taira, K., Colonius, T., MacMynowski, D. G., and Tadmor, G., "Closed-loop control of vortex shedding on a two-dimensional flat-plate airfoil at a low Reynolds number," 2008, AIAA Paper 2008-634.

${ }^{38}$ Ahuja, S. and Rowley, C. W., "Low-dimensional models for feedback stabilization of unstable steady states," 2008, AIAA Paper 2008-553. 